\title{
Efficacy Outcomes in the Phase 3 COMBI-AD Study of Adjuvant Dabrafenib Plus Trametinib vs Placebo in
}

Patients With Stage III BRAFV600E/K-Mutant Melanoma

Georgina V. Long, ${ }^{1}$ Axel Hauschild, ${ }^{2}$ Mario Santinami, ${ }^{3}$ Victoria Atkinson, ${ }^{4}$ Mario Mandalà, ${ }^{5}$ Vanna Chiarion-Sileni, ${ }^{6}$ James Larkin, ${ }^{7}$ Marta Nyakas, ${ }^{8}$ Caroline Dutriaux, ${ }^{9}$ Andrew Haydon, ${ }^{10}$ Caroline Robert, ${ }^{11}$ Laurent Mortier, ${ }^{12}$ Jacob Schachter, ${ }^{13}$ Dirk Schadendorf, ${ }^{14}$ Thierry Lesimple, ${ }^{15}$ Ruth Plummer, ${ }^{16}$ Ran Ji, ${ }^{17}$ Pingkuan Zhang, ${ }^{17}$ Bijoyesh Mookerjee, ${ }^{17}$ Jeff Legos, ${ }^{17}$ Richard Kefford, ${ }^{18}$ Reinhard Dummer, ${ }^{19}$ John M. Kirkwood ${ }^{20}$

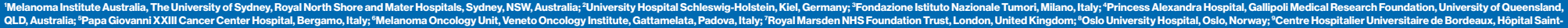

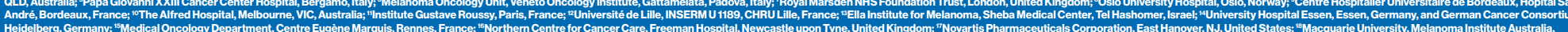

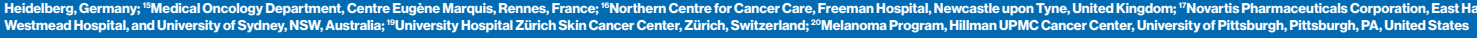

\section{Introduction}

- Surgery alone is often curative for patients with localized melanoma; however, those with regional involvement (stage Ill disease) are at a higher risk for disease progression even with complete surgical resection 1.2

- In phase 3 trials involving patients with previously untreated advanced or metastaticBRAFV600-mutant melanoma dabrafenib plus trametinib combination therapy improved clinical outcomes and was well tolerated ${ }^{3,4}$

- The COMBI-AD study (NCT01682083) is a randomized, double-blind placebo-controlled, phase 3 trial that evaluated the efficacy and safety of dabrafenib plus trametinib combination therapy in patients with completely resected, high-risk, stage III, BRAF V600E/K-mutant melanoma without prior anticancer therapy ${ }^{5}$

Figure 1. Study Rationale ${ }^{3,5-8}$

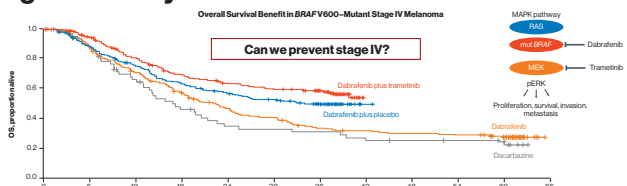

MAPK, mitogen-activated protein kinase; mut, mutated; pERK, phosphorylated extracellular signalegulated kinase

\section{Figure 2. Study Design}

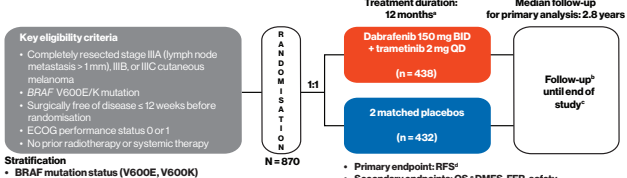

BID, twice daily: DMFS, distant metastasis-free survival; ECOG, Eastern Cooperative Oncology 作 Or until disease recurrence, death, unacceptable toxicity, or withdrawal of consent. -The study will be considered complete and final OS analysis will occur when $\approx 70 \%$ of randomized patients have died or are lost to follow-up.

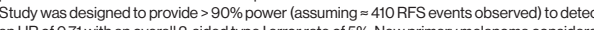
swastobe tex

Figure 3. Primary Analysis: RFS and OS

Median follow-up: 2.8 years
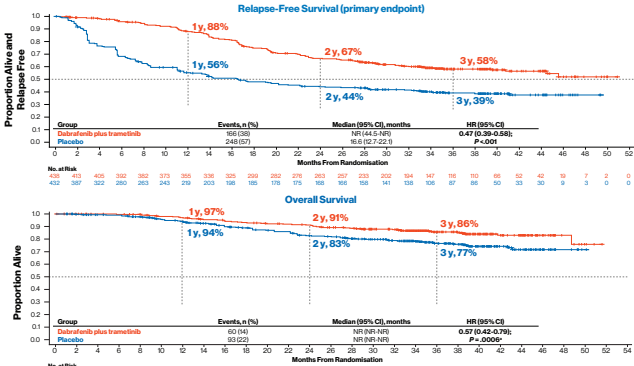

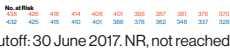

Data cutoff: 30 June 2017.NR, notreached. Prespecified significa

Figure 4. Primary Analysis: RFS by Subgroup

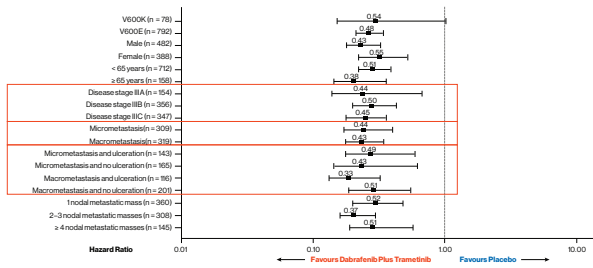

Data cutoff: 30 June 2017.

Figure 5.RFS: Stage IIIA

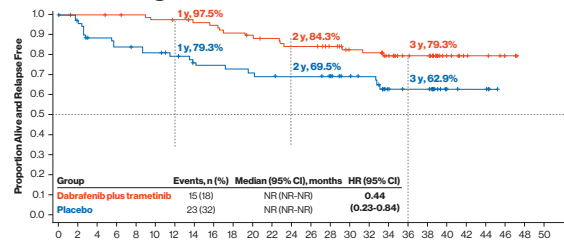

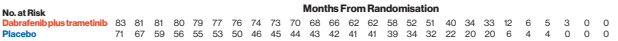

$\mathrm{NR}$, not reached.
Figure 6. RFS: Stage IIIB

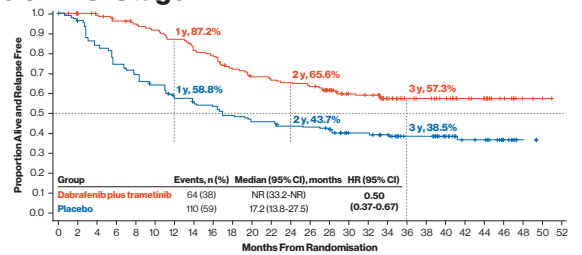

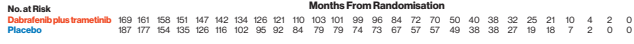

Figure 7.RFS: Stage IIIC

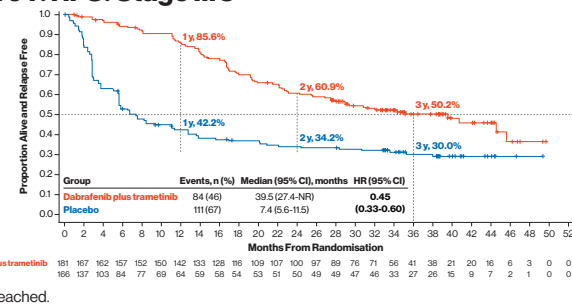

Figure 8. RFS: Stage IIIB and IIIC

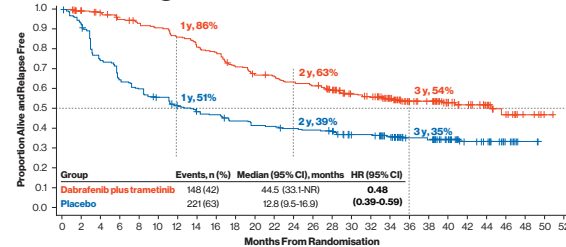

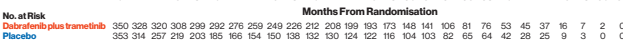

NR, not reached.

Figure 9.RFS: Micrometastases

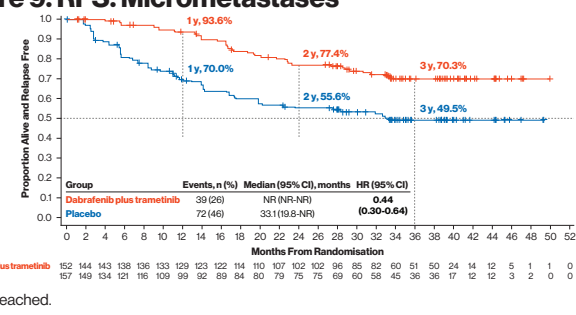

Figure 10. RFS: Macrometastases

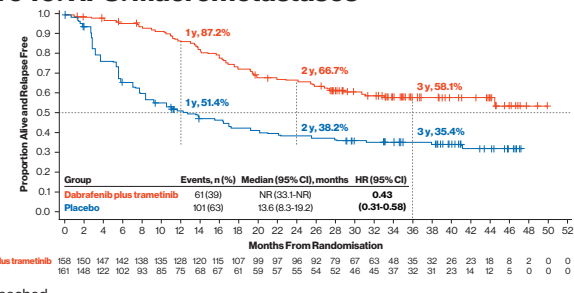

Figure 11. RFS: Without Primary Tumour Ulceration

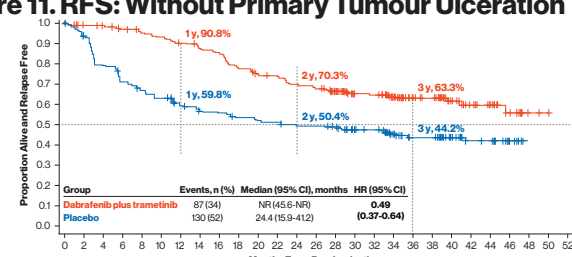

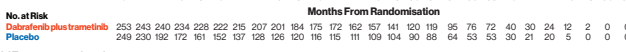

Figure 12. RFS: With Primary Tumour Ulceration

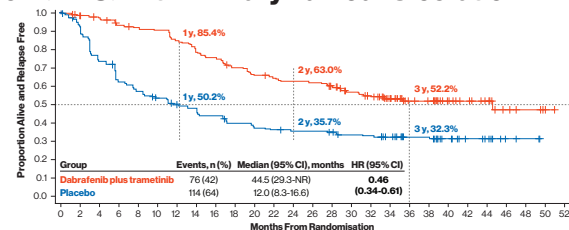

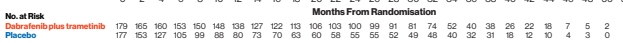
NR, not reached
Table 1. Type of Recurrence at First Recurrence

\begin{tabular}{lcc} 
Type of recurrence, $\mathbf{n}(\%)$ & $\begin{array}{c}\text { Dabrafenib Plus } \\
\text { Trametinib } \\
(\mathbf{n = 1 6 6 )}\end{array}$ & $\begin{array}{c}\text { Placebo } \\
(\mathbf{n}=\mathbf{2 4 8})\end{array}$ \\
\hline Distant recurrence & $103(62)$ & $133(54)$ \\
\hline Local/regional recurrence & $61(37)$ & $114(46)$ \\
\hline Secondary primary melanoma & $7(4)$ & $8(3)$ \\
\hline Death & $3(2)$ & $1(<1)$
\end{tabular}

Table 2. Primary Analysis: Safety Summary

AE Category, $\mathrm{n}(\%)$

Any AE

AEs related to study treatment

Grade $3 / 4 \mathrm{AEs}$ related to study treatment

AnySAE

SAEs related to study treatment

AEs leading to dose interruptio

AEsleading to dose reduction

AEsleading to treatment discontinuation

Fatal AEs related to study drug

$\mathrm{AE}$, adverse event; $\mathrm{SAE}$, serious adverse event.

Most common AEs leading to treatment discontinuation in the a b

- Most common AEs in the dabrafenib plus trametinib arm were pyrexia $(63 \%)$ and fatigue $(47 \%)$

Table 3. Characterisation of Pyrexia Events

\begin{tabular}{lcc} 
& $\begin{array}{c}\text { Pabrafenib Plus } \\
\text { Trametinib } \\
(\mathbf{n}=435)\end{array}$ & $\begin{array}{c}\text { Placebo } \\
(\mathbf{n}=\mathbf{4 3 2})\end{array}$ \\
\hline Patients with pyrexia events, $\mathbf{n}(\%)$ & $292(67)$ & $66(15)$ \\
\hline $\begin{array}{l}\text { Median time to onset of first pyrexia } \\
\text { occurrence (range), days }\end{array}$ & $23(1-28)$ & $53(1-373)$ \\
\hline $\begin{array}{l}\text { Median duration of pyrexia (range), days } \\
\text { Pyrexia event characteristics, } \mathbf{n}(\%)^{\mathbf{a}}\end{array}$ & $3(1-92)$ & $3(1-340)$ \\
$\quad$ Serious adverse event & $71(24)$ & $4(6)$ \\
$\quad$ Grade 3 & $24(8)$ & $2(3)$ \\
$\quad$ Grade 4 & $1(11)$ & 0 \\
\hline $\begin{array}{l}\text { Number of pyrexia occurrences, } \mathbf{n}(\%)^{\mathbf{a}} \\
1\end{array}$ & $83(28)$ & $45(68)$ \\
2 & $57(20)$ & $11(17)$ \\
$\geq 3$ & $152(52)$ & $10(15)$ \\
\hline
\end{tabular}

Percentage based on number of patients with pyrexia

Table 4. Pyrexia Management and Outcome

\begin{tabular}{lcc} 
& $\begin{array}{c}\text { Dabrafenib Plus } \\
\text { Trametinib } \\
(\mathbf{n}=\mathbf{4 3 5})\end{array}$ & $\begin{array}{c}\text { Placebo } \\
(\mathbf{n}=\mathbf{4 3 2})\end{array}$ \\
\hline $\begin{array}{l}\text { Action taken with dabrafenib/trametinib, } \mathbf{n}(\%)^{\mathrm{a}} \\
\text { Drug withdrawn }\end{array}$ & $\begin{array}{c} \\
\text { Do (14)/27(9) }\end{array}$ & - \\
$\quad$ Dose reduced & $86(29) / 18(6)$ & - \\
$\quad$ Drug interrupted & $202(69) / 121(41)$ & - \\
\hline Recovered/resolved, $\mathbf{n}(\%)^{\mathrm{a}}$ & $289(99)$ & $64(97)$ \\
\hline
\end{tabular}

aPercentage based on number of patients with pyrexia.

Table 5. Secondary Malignancies ${ }^{a}$

\begin{tabular}{|c|c|c|}
\hline & $\begin{array}{l}\text { Dabrafenib Plus Trametinib } \\
(\mathrm{n}=\mathbf{4 3 5})\end{array}$ & $\begin{array}{l}\text { Placebo } \\
(n=432)\end{array}$ \\
\hline New primary melanoma & $11(3)$ & $10(2)$ \\
\hline New cuSCC or keratoacanthoma & $8(2)$ & $7(2)$ \\
\hline New basal cell carcinoma & $19(4)$ & $14(3)$ \\
\hline \multirow[t]{2}{*}{ New nonskin malignancies } & $10(2)$ & $4(1)$ \\
\hline & $\begin{array}{l}\text { - Endometrial, } \mathrm{n}=2 \\
\text { - Lung, breast, renal cell, } \\
\text { adenocarcinoma NOS, } \\
\text { chronic myeloid leukaemia, } \\
\text { B-cell lymphoma, lymphoma, } \\
\text { prostate, } \mathrm{n}=1 \text { each }\end{array}$ & $\begin{array}{l}\cdot \text { Colon, } \\
\text { pancreatic, rena } \\
\text { cell, bladder } \\
\text { transitional cell, } \\
\mathrm{n}=1 \text { each }\end{array}$ \\
\hline
\end{tabular}

alncludes events cocuring after randomisation.

\section{Conclusions}

- Dabrafenib plus trametinib reduced the risk of disease recurrence vs placebo in patients with resected stage III, BRAFV600E/K-mutant melanoma; this result was statistically significant and clinically meaningfu - RFS HR, 0.47 ( $95 \% \mathrm{Cl}, 0.39-0.58 ; P<.001)$

- OS HR, 0.57 ( $95 \% \mathrm{Cl}, 0.42-0.79, P=.0006$ [prespecified significance boundary, $P=.000019]$ ]

- RFS benefit was observed in all patient subgroups

- Stage IIIA HR, 0.44; stage IIIB HR, 0.50; stage IIIC HR, 0.45

- Micrometastases HR, 0.44; macrometastases HR, 0.43

- Nonulcerated HR, 0.49; ulcerated HR, 0.46

- Although pyrexia was the most common AE, it was well characterised and manageable

Dabrafenib plus trametinib represents a significant advance for the adjuvant treatment of stage III BRAFV600-mutant melanoma

\section{Acknowledgements}

Wealso thank study site staff, additional investigators, and others for their contributions
This study was sponsored by GlaxoSmithKline; dabrafenib and trametinibare assets of Novartis AG as of 2 March 2015

\section{References}

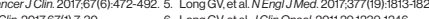

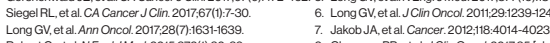

. Poster Presentation at the Winter Clinical Dermatology Conference; January 12-17, 2018; Kaanapali, HI.
This was previously presented at the 9th World Congress of Melanom/14th International Congress of This was previously presented at the
the Society for Melanoma Research 